\title{
Reflections on Communication Difficulties Related to Covid-19
}

\author{
Suzanne Ekelund \\ Retired Principal Specialist, Clinical Biochemist, M. Sci. \\ Roedovre, Denmark \\ Email: suzanne.ekelund [AT] gmail.com
}

\begin{abstract}
This paper describes some of the concept which are often miscommunicated between scientists, politicians and laypeople. Scientists are advised to take a more active role in explaining relevant concepts so that they can be understood by laypeople and thus facilitate better discussions.
\end{abstract}

Keywords- covid-19, communication difficulties, uncertainty, laypeople explanations

\section{INTRODUCTION}

It sometimes feels as if the society has been split in many fractions during the covid-19 pandemic. We have people doing what the authorities tell them to do, anti-vaxxers, lockdown-sceptics, etc. The main problem is not that people have different opinions but the increasing lack of respect and understanding of people thinking and choosing differently from yourself. A contributing factor to this situation is communication problems.

An important triangle in the communication problems is the triangle made up by the scientists, the politicians and the public (laypeople). There are differences of opinion and communication problems between these three groups and also within the specific groups. Journalists and media also play a role in this when they try to mediate the opinions of especially the scientists and the politicians. However, the focus of this paper is communication from scientists to other groups and between different groups of scientists.

A few key problems that scientists should make an effort to explain better are addressed.

\section{SOME KEY CONCEPTS}

The communication and discussions related to covid-19 would benefit from a common understanding between the scientists, the politicians and the public (laypeople) of at least a few key concepts and problems. The list is not and cannot be comprehensive. Focus is on communication from scientists to other groups and between different groups of scientists.

\subsection{Evidence Based}

A major problem is to make people understand that life (medical) sciences are evidence-based sciences and not exact sciences like mathematics. When a treatment is evidence based it means that it is supported by a large amount of scientific research [1]. It is not as in mathematics where $2+2$ make 4 and research will not change that. However, in life sciences research can change what is considered evidence based. This can also be difficult to understand for statisticians who sometimes think that you can compare human beings in a population to coins in a jar and that you can do experiments and statistics without taking into consideration that you are dealing with human beings who are always different from one another, even identical twins are different from one another. All this means that you have to have large studies to conclude anything with a statistical significance because you have to make it likely that you have groups representing the average population. And sometimes the studies you would like to perform are unethical and you have to design a study, which might only give you an indication of what you would like to know.

\subsection{Average Life Expectancy}

What is "average life expectancy" or just "life expectancy"? In short, the term refers to the number of years a person can expect to live. By definition, life expectancy is an estimate of the average age that members of a particular population group will be when they die [2]. However, you can calculate this in different ways. You can calculate the average life length of individuals born in a given year (called the cohort life expectancy) or you can estimate the average length of life for a whole population and assumed all are exposed, from birth through death, to the mortality rates observed at one particular period - commonly a year. It typically reflects the mortality pattern as it is right now. As this definition is used by most international organizations, including the United Nations, when reporting life expectancy figures, I will use it in the following. 
Many people think that a person close to the life expectancy age has a great likelihood of dying soon. This is wrong. A person close to the life expectancy age has a great likelihood of living for several more years. Life expectancy is an average. Included in this average are infant deaths, deaths by accident, suicide, drug overdose etc. These contributions lower the average. When you reach the average the likelihood of dying from these mentioned causes is small. In Sweden where there were no corona restrictions in the beginning of the pandemic the life expectancy has decreased, for men by 11 months and for women by six months [3]. This makes many people think "OK, some men died 11 months before they otherwise would have died". This is not true. When the average decreases it means that those who died from covid-19 died several years before they would otherwise have died. The reason is that the fraction of people dying from covid-19 is still only a small fraction of all people dying in Sweden and these people dying from covid-19 were able to lower the average for all dying.

\subsection{Uncertainties}

The public are given a lot of numbers to keep them informed about the progression of the pandemic. The public may have an idea about what the concepts and numbers represent but who explains about the uncertainties associated with these numbers?

The uncertainties come mainly from lack of exact information. False test results and test strategy may contribute to this. There are numerous concepts presented to the interested public. As the estimated numbers for several of these concepts are also used by politicians and health care authorities to decide potential restrictions and emergency preparedness it is important to have an idea about the size of the uncertainties involved in the estimates.

Examples of relevant concepts with non-negligible uncertainties are:

- $\quad$ Base Rate

- Confirmed base rate

- Positivity rate

- Transmission rate

\section{Base rate and confirmed base rate:}

To get an idea about the impact of a pandemic you would like to know the base rate, that is how many in the society are right now infected. To get a precise number for that you would have to test all people in the society and you need tests, which are $100 \%$ accurate. That is of course not possible. Therefore, confirmed base rate is used to estimate the base rate. A confirmed case is a person who has been diagnosed as having the disease, that is covid-19. From the number of confirmed cases experts estimate the total number of cases and that estimate is the base rate. However, these estimates can be optimistic or pessimistic. In Denmark Statens Serum Institut (part of the Danish Ministry of Health) has been criticized for too pessimistic estimates of base rate increasing the assumed base rate with a factor of 4 [4].

In addition, the definition of the disease, the tests used and the test strategy add great uncertainties.

The current definition is that you are infected with covid-19 if you have a positive result with a so-called PCR-test. This means that during the pandemic the healthcare system has abandoned the important rule of interpreting all laboratory results in the clinical context [5], a rule, which means that a single laboratory result cannot alone determine the diagnosis. If you get a negative result in a patient who shows all signs of having the disease tested for, you should either repeat the test or do additional tests/assessments to clarify the situation. The same is the case if you get a positive result in a patient who shows no signs of having the disease tested for.

No test always gives the correct result [5]. You may get false negative results that is negative results in patients actually having the disease tested for, and you may get false positive results that is positive results in patients not having the disease tested for. The fractions of respectively false negative and false positive results are specific for a specific test from a specific manufacturer. If the test you use gives $10 \%$ false negative results in persons with covid-19, then one in ten sick persons will get a false negative result, keep living as usual and infect friends and family.

Finally test strategy has an influence on how well you can trust the results. The WHO advised in the beginning of the pandemic countries to "test, test, test" [6]. When you use that strategy, you will test a lot of healthy persons. If the test you use gives $0.1 \%$ false positive results, one in thousands of these healthy persons will get a false positive result [5].

Table 1. Example of test results when a population of 100,000 healthy and 1000 sick persons are tested. The test used gives $10 \%$ false negative results in sick persons and $0.1 \%$ false positive results in healthy persons.

\begin{tabular}{|l|c|c|c|}
\hline Test result & & Positive & Negative \\
\hline Healthy persons, \# & 100,000 & 100 & 99,900 \\
\hline Sick Persons, \# & 1,000 & 900 & 100 \\
\hline Total tested, \# & 101,000 & 1,000 & 100,000 \\
\hline
\end{tabular}


The example in table 1 shows you that if you test 101,000 persons of whom 1,000 are sick then you will with a typical PCR covid-19 test get 1,000 positive results. However, 100, equal to $10 \%$ of the results, will be false positive results in healthy persons, while 100 negative results will be false negative results in sick persons. As we will see below in figure 1 the fraction of false positive results depends on how many healthy, we test.

So, if you want to make people understand and trust an estimate of base rate you need to explain about the uncertainties and the models, you use for your estimation. You could calculate best, worst and most likely scenario. In situations where it has been shown that your former estimates were far from the truth you need to explain what you have done to make your current estimates more reliable. This may be a huge task but it will be worth it because it will make the public more confident with your estimates.

\section{Positivity rate}

The positivity rate is often mentioned as a measure of how well the pandemic is under control. The positivity rate is the percentage of all covid-19 tests performed that are actually positive. Usually, the opinion is that the lower it is, the better.

In the example in table 1 the positivity rate is calculated as 1,000/101,000, meaning that it is $0.99 \%$. As can be seen in figure 1 the percentage of true positive results for a specific test is a function of positivity rate. The higher the positivity rate the higher the percentage of true positive results.

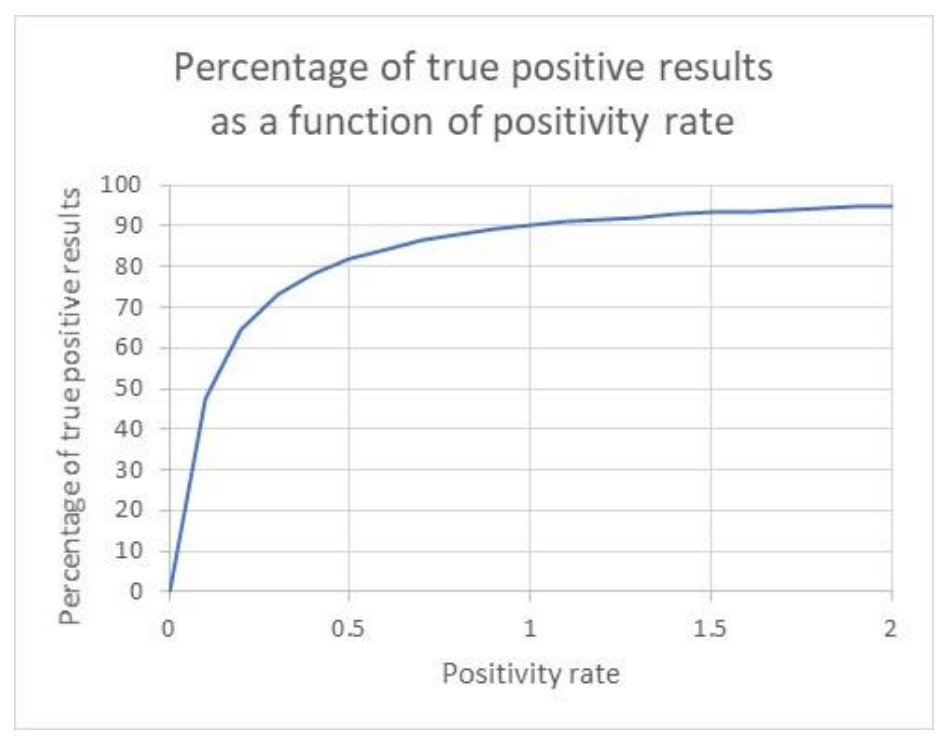

Figure 1. Percentage of true positive results as a function of positivity rate. Calculated for a test with $10 \%$ false negative results in sick persons and $0.1 \%$ false positive results in healthy persons.

Now why should we think that a low positivity rate is good? A low positivity rate just means that a lot of healthy persons have been tested because the authorities say "test, test, test" [6] and maybe some of these tested healthy persons need a certificate with a negative test less than 2 or 3 days old to get into some social event.

The truth is the lower the positivity rate, the lower the percentage of true positive results and thus the higher the number of false positive results, meaning time and money wasted on testing healthy persons. How do we explain that we should not focus on positivity rate in a way so that everyone understands it?

\section{Transmission rate}

The transmission rate tells us how many new persons an infected person on average will infect. This measure is used in epidemiology models to forecast the spread of infection and the severity of consequences [7]. Values greater than 1.0 implies an increase in the number of cases, while values lower than 1.0 implies a decrease in the number of cases. The number which epidemiologists can use to estimate transmission rate are confirmed cases. As we saw above the number of confirmed cases has uncertainties based on tests used and test strategy. It makes sense to calculate transmission rates on regional basis but that also introduces uncertainties regards the infected persons whereabouts. Where did they get infected - on a trip to another region or at home? The estimated transmission rates should of course be published but could we find a suitable disclaimer to let people know that there are uncertainties?

\subsection{Long covid-19}

When the pandemic had lasted for some months, it became obvious that some persons who had had covid-19 and recovered, had not recovered totally. They had what we now call long covid-19. Some have had it for more than a year 
now. The symptoms can be various and we still try to define what long covid-19 is. Reviews on the topic are frequently made. Late May 2021 two reviews were made based on respectively 65 [8] and 194 [9] references. This illustrates that there are many publications on the topic. However, still many laypeople do not accept that long covid-19 is a problem so we definitely need to increase the knowledge of long covid-19 in the population by writing about it in a way, which is easy to understand.

\subsection{Vaccines}

The three main approaches to making a vaccine are based on respectively, the whole virus/bacterium, parts of the virus/bacterium that will trigger the immune system or parts of the genetic material (nucleic acids) [10]. As the latter is new it should be better explained to the public. All pros and cons should be presented. Even when it comes to how to preserve the vaccine. It can be seen as a disadvantages that nucleic acid vaccines have to be kept at very low temperatures for storage but the advantage is that it does not need toxic components, like mercury, or formaldehyde, which many people are allergic to, for preservation.

There is also a need to explain the various degrees of approval: Standard marketing authorization, conditional marketing authorization and emergency use authorization. What are the differences?

To be approved drugs/vaccines have to go through three clinical trial phases:

- In phase 1 safety, tolerability and toxicity at different doses is tested in 20-100 healthy volunteers.

- In phase 2 efficacy and determination of optimal dose is tested in up to several hundred persons with the relevant disease

- Phase 3 typically involves 300 to 3,000 persons from patient populations for which the drug/vaccine is eventually intended to be used. Participants are assigned to receive either the drug/vaccine being evaluated or to a control group that receives either the current standard of care treatment or a placebo (a substance that has no therapeutic effect).

If phase 3 is successful the manufacturer can submit data with a request for authorization. It is the extent of phase 3 that differs between the various authorizations.

\section{Conditional marketing authorization}

A conditional marketing authorization may be granted for drugs and vaccines that address unmet medical needs [11]. The authorization is based on less comprehensive clinical data than normally required, where the benefit of immediate availability of the drug/vaccine outweighs the risk inherent in the fact that additional data are still required. The drug/vaccines are eligible if they are intended for seriously debilitating or life-threatening diseases or if they are intended for a public health emergency (e.g. a pandemic). Conditional marketing authorizations are valid for one year and can be renewed annually. Once a conditional marketing authorization has been granted, the marketing authorization holder must fulfil specific obligations within defined timelines. These could include completing ongoing or new studies or collecting additional data to confirm the drug/vaccine's benefit-risk balance remains positive.

\section{Standard marketing authorization}

The conditional marketing authorization can be converted into a standard marketing authorization (no longer subject to specific obligations) once the marketing authorization holder fulfils the obligations imposed and the complete data confirm that the drug/vaccine's benefits continue to outweigh its risks.

\section{Emergency use authorization}

An Emergency Use Authorization (EUA) can be granted in special situations like during public health emergencies [12]. Under an EUA, the use of unapproved medical products, or unapproved uses of approved medical products may be allowed in an emergency if there are no adequate, approved, and available alternatives. An EUA requires adequate information to ensure quality and consistency, including evaluation of the chemistry, manufacturing, and controls information. The known and potential benefits must outweigh the known and potential risks of the vaccine. The EUA may be based on a final analysis of a phase 3 clinical efficacy trial or an interim analysis of such trial, i.e., an analysis performed before the planned end of the trial once the data have met the pre-specified success criteria for the study's primary efficacy endpoint.

An EUA includes an expectation that the follow-up for at least half of vaccine recipients in the phase 3 clinical trials is at least 2 months after completion of the final vaccination. The phase 3 shall include well over 3,000 vaccine recipients, and a high proportion of participants must have been followed for serious adverse events and adverse events of special interest for at least one month after completion of final vaccination. 


\section{CONCLUSION}

As there seems to be a communication gap in society, my advice to fellow scientists is to write readers' letters to your newspapers, posts on social media etc. with explanations that laypeople may understand. Talk to your friends and explain the different topics to them. You don't have to agree on what is the best way to tackle the situation. However, knowledge is a better platform for discussions and a better understanding may hopefully avoid some of the conspiracy theories, which unfortunately have also been circulating during the pandemic.

If or when we have a new pandemic this will still be a good approach. The more information we have on a subject the better discussions we can have about it.

\section{REFERENCES}

[1] Cambridge Dictionary (accessed June 5th 2021 - https://dictionary.cambridge.org/dictionary/english/evidence-based

[2] Our World Data website (accessed June 7th 2021 - https://ourworldindata.org/life-expectancy-how-is-it-calculatedand-how-should-it-be-interpreted

[3] Swedish Radio March 4th 2021 quoting Statistics Sweden - https://sverigesradio.se/artikel/covid-19-sankerlivslangden-och-hojer-pensionen

[4] News from Danish Broadcasting Corporation. May 6th 2020 - https://www.dr.dk/nyheder/indland/ny-kritik-afcorona-beregninger-moerketal-kan-vaere-meget-mindre

[5] Ekelund SL: False Covid-19 Test Results - Could We Do Things Better? A Personal Opinion. Asian Journal of Pharmacy, Nursing and Medical Sciences, 2020; 8(5). https://doi.org/10.24203/ajpnms.v8i5.6349

[6] Reuters March 16th 2020 - https://www.reuters.com/article/us-healthcare-coronavirus-who-idUSKBN2132S4

[7] Rode DC, Fischbeck PS. On Ambiguity Reduction and the Role of Decision Analysis during the Pandemic. Risk Analysis, 2021; 41(5): 721-730. DOI: 10.1111/risa.13705

[8] Nasserie T, Hittle M, Goodman SN. Assessment of the Frequency and Variety of Persistent Symptoms Among Patients With COVID-19: A Systematic Review. JAMA Netw Open. 2021 May 3; 4(5): e2111417. doi: 10.1001/jamanetworkopen.2021.11417

[9] Yong SJ. Long COVID or post-COVID-19 syndrome: putative pathophysiology, risk factors, and treatments. Infect Dis (Lond). 2021 May 22:1-18. doi: 10.1080/23744235.2021.1924397

[10] WHO website. The different types of COVID-19 vaccines. https://www.who.int/news-room/featurestories/detail/the-race-for-a-covid-19-vaccine-explained

[11] European Medicines Agency website. Conditional marketing authorization. (Accessed June 14th 2021 https://www.ema.europa.eu/en/human-regulatory/marketing-authorisation/conditional-marketing-authorisation

[12]FDA website. Emergency Use Authorization for Vaccines Explained. (Accessed June 14th 2021 https://www.fda.gov/vaccines-blood-biologics/vaccines/emergency-use-authorization-vaccines-explained 